\title{
Troxerutin and Cerebroprotein Hydrolysate Injection Protects Neurovascular Units from Oxygen-Glucose Deprivation and Reoxygenation-Induced Injury In Vitro
}

\author{
Hóngyi Zhào, ${ }^{1,2}$ Yu Liu, ${ }^{2}$ Jing Zeng,, Dandan Li, ${ }^{1}$ Weiwei Zhang, ${ }^{1}$ and Yonghua Huang ${ }^{1}$ \\ ${ }^{1}$ Department of Neurology, PLA Army General Hospital, 5 Nanmencang Dongsishitiao Street, Beijing 100007, China \\ ${ }^{2}$ Department of Neurology, No. 261 Hospital of PLA, 116 Zaojiatun Shangzhuang, Beijing 100094, China \\ Correspondence should be addressed to Yonghua Huang; huangyonghua2017@126.com
}

Received 24 November 2017; Accepted 2 April 2018; Published 2 May 2018

Academic Editor: Kuo-Tong Liou

Copyright (c) 2018 Hóngyi Zhào et al. This is an open access article distributed under the Creative Commons Attribution License, which permits unrestricted use, distribution, and reproduction in any medium, provided the original work is properly cited.

Cerebral ischemia/reperfusion (I/R) injury involves complex events of cellular and molecular processes. Previous studies suggest that a neurovascular unit (NVU) acts as an intricate network to maintain the neuronal homeostatic microenvironment. The present study established an NVU model for oxygen-glucose deprivation and reoxygenation (OGD/R) damage, trying to target the major components of the NVU using a coculture of rat neurons, astrocytes, and rat brain microvascular endothelial cells (rBMECs) to investigate the therapeutic effects of troxerutin and cerebroprotein hydrolysate injections (TCHis). The study observed that OGD/R downregulated the expressions of GAP-43, Claudin-5, and AQP-4 obviously detected by Western blotting and immunocytochemical analysis, respectively, while TCHi ameliorated the effect of OGD/R significantly. Meanwhile, TCHi alleviated the abnormalities of ultrastructure of neurons and rBMECs induced by OGD/R. Furthermore, both levels of inflammatory cytokines (IL-1 $\beta$, IL-6, and TNF- $\alpha$ ) and cell adhesion molecules (VCAM-1 and ICAM-1) detected by ELISA in NVU supernatant were found elevated significantly through OGD/R, but TCHi ameliorated the trend. In addition, TCHi also mitigated the increase of proapoptotic factors (Bax, p53, and caspase-3) induced by OGD/R in NVU model statistically. All these findings demonstrated that TCHis played a protective role, which was reflected in anti-inflammation, antiapoptosis, and blood-brain barrier maintenance. The results of the study concluded that the NVU is an ideal target and TCHi acts as a neuroprotective agent against cerebral I/R injuries.

\section{Introduction}

Cerebral ischemia/reperfusion (I/R) injury is a complex pathological process in the nervous system, resulting in high disability and mortality worldwide, with significant clinical and socioeconomic impacts [1]. The complex pathobiological mechanisms of this medical problem include inflammation, apoptosis, oxidative damage, and ionic imbalances [2]. Although reperfusion after ischemia is essential for cell survival, it may have numerous negative consequences such as microvascular damage, cell dysfunction, and cell death. It can attract leukocytes and cause the release of several proinflammatory mediators and the induction of microglia and macrophages [3]. Excessive neuroinflammation can increase brain damage and bring about many secondary complications that influence stroke outcome; therefore, anti-inflammation is considered a target for ischemic stroke.

A neurovascular unit (NVU), consisting mainly of microvessels, astrocytes, neurons, extracellular matrix, and other types of gliocytes, is defined as a complete functional and structural unit of the brain [4]. Not only do neurons suffer from strokes, but the microvasculature and gliocytes are also involved [5]. Consequently, protecting different cell types simultaneously in the NVU is necessary for I/R injury therapy [6].

Troxerutin, a naturally occurring flavonoid, is known mainly because of its anti-inflammatory, antioxidative, antithrombotic, antineoplastic, and antiapoptotic activities 
$[7,8]$. Cerebroprotein hydrolysate (with abundant bioactive peptides) was found to facilitate the distribution of troxerutin and had a positive synergistic effect with troxerutin against acute ischemic stroke [9]. The present study aimed to investigate the protective effects of troxerutin and cerebroprotein hydrolysate injections (TCHis) on oxygen-glucose deprivation and reoxygenation- (OGD/R-) inducing NVU dysfunction and the possible mechanism.

\section{Methods}

2.1. Animals. Adult Wistar rats (3 months old) were purchased from Peking Vital River Laboratory Animal Ltd. Three female rats were mated with one male rat in each cage, and the pregnant females were kept individually. The rat pups were used for further experiments. All experiments were performed in accordance with China's Guidelines for Care and Use of Laboratory Animals.

2.2. Primary Cell Cultures. Primary cells were extracted from the rat pups and routinely cultured in conditioned incubators $\left(37^{\circ} \mathrm{C} / 5 \% \mathrm{CO}_{2}\right)$. The isolation procedure was performed according to the methods used by Xue et al. (2013) [10] and Wang et al. (2015) [11].

Primary cortical neurons $(\mathrm{N})$ were prepared from Wistar newborn rats (less than $24 \mathrm{~h}$ ). In brief, the cerebral cortex was digested with $0.125 \%$ trypsin for $10 \mathrm{~min}$ at $37^{\circ} \mathrm{C}$ and the cell suspension was passed through a $75 \mu \mathrm{m}$ pore filter. Cells were harvested and seeded on poly-D-lysine (Sigma Aldrich, MO, USA) precoated plates in Neurobasal Medium (Invitrogen, CA, US) containing 2\% B27 supplement (Invitrogen), 1\% penicillin-streptomycin, and $2 \mathrm{mM}$ L-glutamine. Experiments were performed for 8 days in vitro.

Primary astrocytes (A) were extracted from 1- to 2-dayold rat pups, as described previously with a few modifications (Saini MG et al., 2011). In brief, the cerebral cortex was digested with $0.125 \%$ trypsin for $10 \mathrm{~min}$ at $37^{\circ} \mathrm{C}$, and the cell suspension was then passed through a $75 \mu \mathrm{m}$ pore filter. Cells were seeded in the DMEM/F12 medium containing $10 \%$ fetal bovine serum (FBS) (NQBB, Australia) and $1 \%$ penicillin-streptomycin. After 7-10 days, the cultures were shaken at $37^{\circ} \mathrm{C}$ at a speed of $260 \mathrm{rpm}$ for $16 \mathrm{~h}$ to remove contaminating microglia and oligodendrocytes. The third passage of astrocytes was used for the following study.

Primary brain microvascular endothelial cell (rBMEC) cultures were established from 7-day-old rat pup brain tissues (B), which were extracted and homogenized with type II collagenase/DNaseI (Sigma) for $1 \mathrm{~h}$. Microvessels were separated after density centrifugation (spun at $1000 \mathrm{~g} / \mathrm{min}$ for $20 \mathrm{~min}$ ) in $20 \%$ bovine serum albumin at $4^{\circ} \mathrm{C}$. The microvessels were then digested using a collagenase/dispase solution (Roche Applied Science, Mannheim, Germany) containing DNaseI for $1 \mathrm{~h}$ and suspended in a DMEM high-glucose medium containing $20 \%$ FBS, $10 \mathrm{ng} / \mathrm{mL}$ basic fibroblast growth factor, $30 \mathrm{U} / \mathrm{mL}$ heparin, $2 \mathrm{mM}$ glutamine, and $1 \%$ penicillin-streptomycin. Cells were then seeded into gelatin
(1\%) coated flasks, and the third passage of rBMECs was used in this study.

2.3. Establishment of NVU In Vitro. The NVU model was established according to the previous report using purified normal morphological cells (Figure 1) [12]. Briefly, after the neurons had grown in a six-well culture plate with a density of $0.5 \times 10^{5}$ cells $/ \mathrm{cm}^{2}$ for 2 days, the purified astrocytes with $1.5 \times 10^{5}$ cells $/ \mathrm{cm}^{2}$ were seeded on the outer side of the insert membrane, which faced the bottom of the well. After $4 \mathrm{~h}$ for astrocyte adhering, the insert was placed into the well with neurons. Two days later, rBMECs $\left(1.0 \times 10^{5}\right.$ cells $\left./ \mathrm{cm}^{2}\right)$ were seeded in the inner side of the insert membrane. After being cocultured for 3-5 days, the NVU model was prepared for the following experiments (Figures 1 and 2).

2.4. Four-Hour Leakage Detection. Blood-brain barrier (BBB) permeability was evaluated by performing a 4 -hour leakage experiment. After 3 days of coculture, the upper inserts were filled with the medium, while the level of the medium in the plates was maintained $0.5 \mathrm{~cm}$ lower than the level of the medium in the upper inserts. Inserts with no cells were used as a control. After $4 \mathrm{~h}$, changes in the level of the medium in the top inserts were observed (Figure 2).

\subsection{Establishment of $\mathrm{OGD} / \mathrm{R}$ Damaging $\mathrm{NVU}$ and TCHi} Treatment. The prepared NVU cells were cultured in a conditional medium [glucose-free, $98.5 \mathrm{mM} \mathrm{NaCl}, 10.0 \mathrm{mM}$ $\mathrm{KCl}, 1.2 \mathrm{mM} \mathrm{MgSO}_{4}, 0.9 \mathrm{mM} \mathrm{Na} \mathrm{HPO}_{4}, 6.0 \mathrm{mM} \mathrm{NaHCO}$, $1.8 \mathrm{mM} \mathrm{CaCl}_{2}, 40 \mathrm{mM}$ sodium lactate, and $20 \mathrm{mM}$ HEPES (Sigma) at a $\mathrm{pH}$ of 6.8] and placed in an anaerobic incubator (BINDER CB150, Germany) with conditions of $5 \% \mathrm{CO}_{2}, 0.2 \%$ $\mathrm{O}_{2}$, and $37^{\circ} \mathrm{C}$ for $2 \mathrm{~h}$ (named as Model group). Then, cultures were switched to completely normal conditions with TCHi at concentrations of $10 \mu \mathrm{M}, 100 \mu \mathrm{M}$, or $1000 \mu \mathrm{M}$ for $2 \mathrm{~h}$ [TCHi, Jilin Sihuan Pharmaceutical Co. Ltd., was dissolved in sterile phosphate-buffered saline (PBS)]. Cells cultured in media with PBS under normoxic conditions were used as a control (named as CK group).

2.6. Transmission Electron Microscopy. The method of using the transmission electron microscope (TEM) was similar to what Liu et al. (2011) [13] and Garbuzova-Davis et al. (2007) [14] mentioned in their study. The cocultures were postfixed in $1 \%$ osmium tetroxide (Electron Microscopy Sciences, Inc., PA, USA) in $0.1 \mathrm{M} \mathrm{PB}$ for $1 \mathrm{~h}$ at room temperature. Following osmication, they were dehydrated in a graded series of acetone dilutions (30\%, 50\%, 70\%, and 95\% acetone in water), allowing $10 \mathrm{~min}$ for each change. Three $10 \mathrm{~min}$ changes in $100 \%$ acetone were made, and the cocultures were transferred to a 50:50 mix of acetone: LX112 epoxy resin embedding mix (Ladd Research Industries, Burlington, VT). Subsequently, the cocultures were infiltrated with this mix for $1 \mathrm{~h}$ under vacuum and were transferred to a 100\% LX112 embedding mix and infiltrated for $1 \mathrm{~h}$ on a rotator. Two more $1 \mathrm{~h}$ infiltration steps were performed with fresh changes of the embedding mixture. Cocultures were further infiltrated in a fresh embedding medium at $4^{\circ} \mathrm{C}$ overnight. The following 


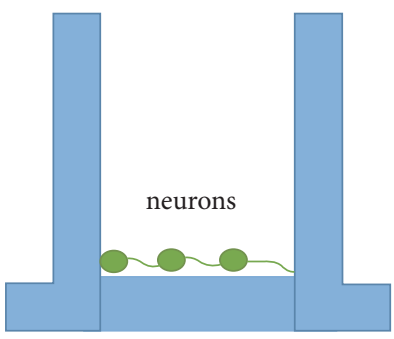

Neurons were cultured on the bottom of transwell filter

(a)

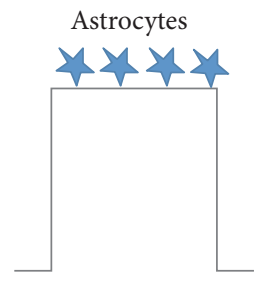

Astrocytes were seeded on the opposite side of transwell filter of the inserts

(b)

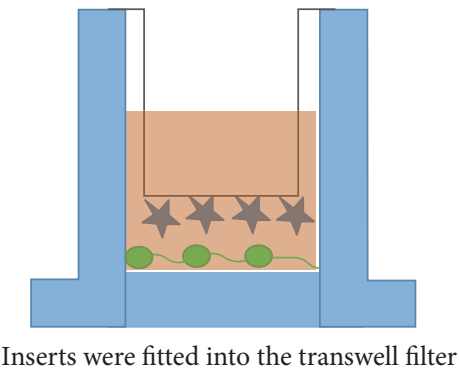

(c)

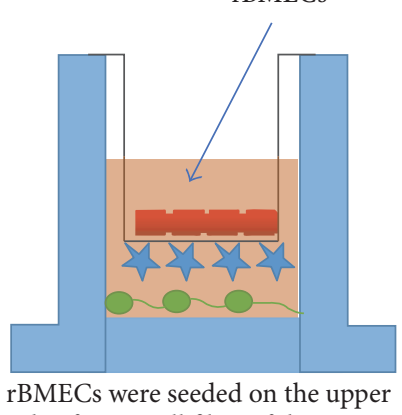

side of transwell filter of the inserts

(d)

FIGURE 1: In vitro NVU model. Sequential steps are shown in (a), (b), (c), and (d).

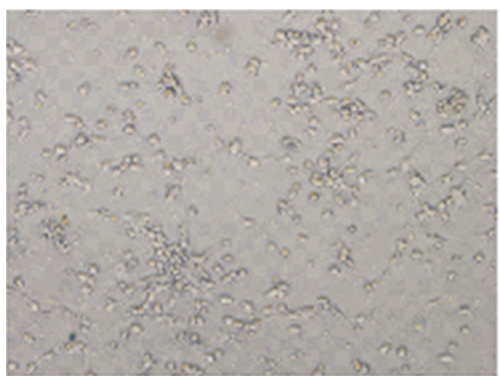

(a)

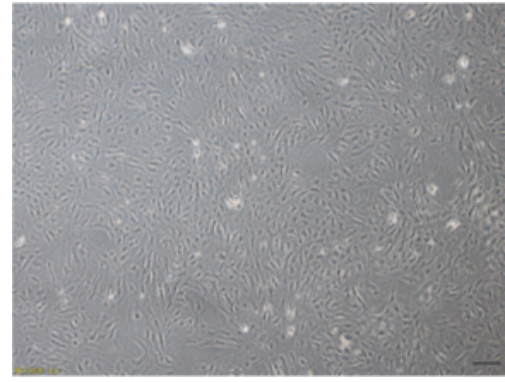

(b)

TEER Value

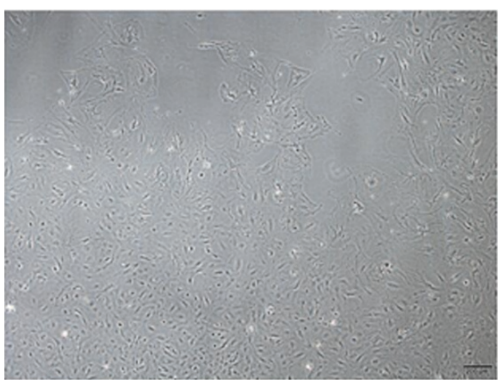

(c)

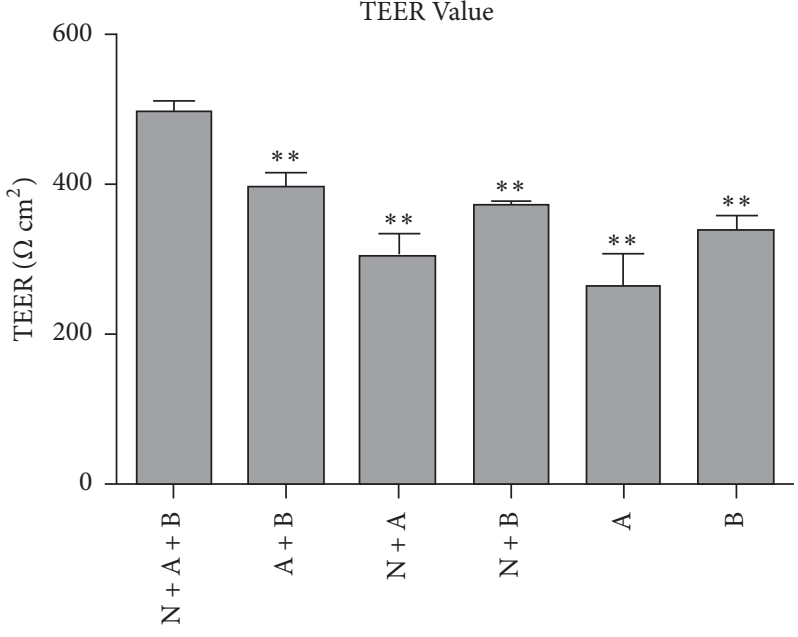

(d)

FIGURE 2: Morphologies of the three types of cells in NVU models. (a), (b), and (c) are neurons, rBMECs, and astrocytes in visible light under the fluorescent inverted microscope, respectively. (d) reveals the TEER values of different culture models, which indicate that the BBB of the NVU model is intact. 


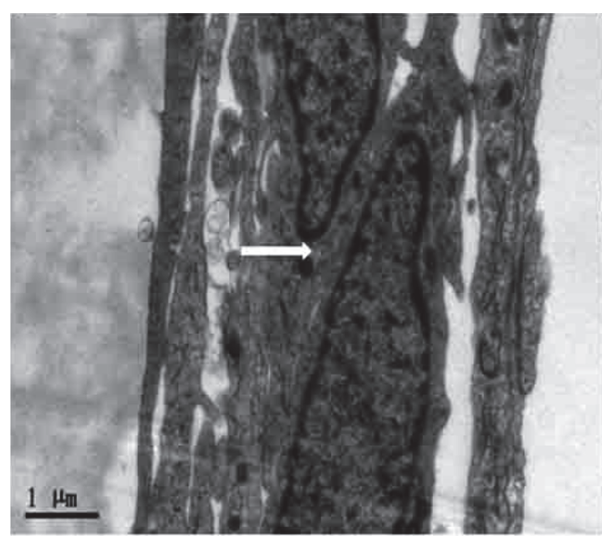

(a)

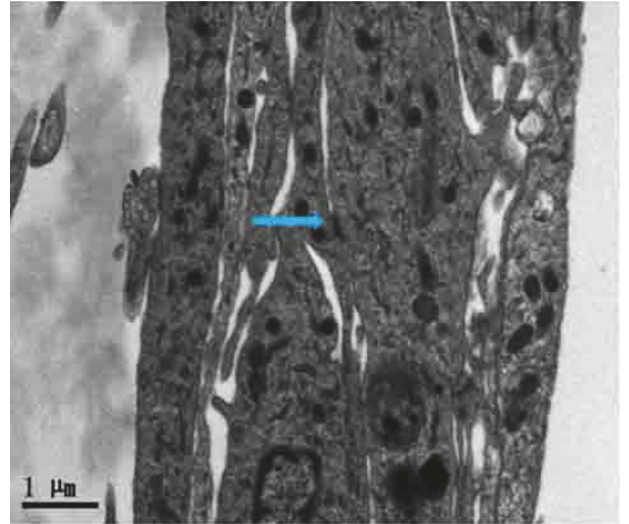

(b)

FIGURE 3: TEM showed that the BBB of rBMECs had intact and continuous tight junctions (white arrow) and desmosomes (blue arrow).

day, the tissues were infiltrated in two additional changes of embedding medium at room temperature, $4 \mathrm{~h}$ per change, and then embedded in a fresh change of resin in tissue capsules. The blocks were polymerized at $70^{\circ} \mathrm{C}$ in an oven overnight and then trimmed and sectioned with a diamond knife on a Reichert Ultracut E ultramicrotome (Leica Microsystems, Inc., IL, USA). Thick sections cut at $0.35 \mathrm{~m}$ were placed on glass slides and stained with $1 \%$ toluidine blue. Thin sections were cut at $80-90 \mathrm{~nm}$, placed on copper grids, and stained with uranyl acetate and lead citrate. The sections were examined and photographed with a Philips CM10 TEM (FEI, Inc., OR, USA) at $60 \mathrm{kV}$. The arrangement of parenchymal cells and cerebral microvessel endothelial cells on the Transwell filter was detected in the cocultures.

2.7. Western Blotting. Cells in the NVU were individually scraped down and lysed on ice for $10 \mathrm{~min}$. After centrifugation $\left(13,000 \mathrm{~g}, 4^{\circ} \mathrm{C}\right)$, the resulting supernatant was saved as the cytoplasmic extract sample and the nuclear pellet was prepared for a nuclear extract sample. The samples were separated by $10 \%$ sodium dodecyl sulfate-polyacrylamide gel electrophoresis (SDS-PAGE) (P0012A, Beyotime, China) and then transferred to polyvinylidene difluoride membranes. The membranes were blocked for $1 \mathrm{~h}$ and incubated overnight at $4^{\circ} \mathrm{C}$ with the following antibodies: rabbit polyclonal antibody against GAP-43 (1:1000, 8945S, CST, China), rabbit polyclonal against AQP-4 (1:1000, ab31721, Abcam, China), rabbit polyclonal antibody against Claudin5 (1:1000, ABT45, Millipore, China), and mouse polyclonal antibody against Tubulin $(1: 200$, sc-5286, Santa Cruz, China). Membranes were incubated with a secondary goat anti-rabbit/mouse antibody (1:3,000, Service, China) for $1 \mathrm{~h}$ at $37^{\circ} \mathrm{C}$. Immunoreactive bands were observed using the ECL detection system (Bio-Rad, Beijing, China).

2.8. Immunocytochemical Analysis. For immunocytochemical analysis, cells were blocked with $10 \%$ normal goat serum in PBS containing $0.1 \%$ Triton X-100 (Sigma) for $30 \mathrm{~min}$ before incubation with primary antibodies for $18 \mathrm{~h}$ at $4^{\circ} \mathrm{C}$ : Rabbit polyclonal antibody against Anti-GAP-43 (1:100, 8945S, CST, China), rabbit polyclonal against AQP-4 (1:100, ab31721, Abcam, China), rabbit polyclonal antibody against claudin-5 (1:100, ABT45, Millipore, China), goat monoclonal antibody against caspase-3 (1:100, EB07286, Everest Biotech, UK), mouse monoclonal antibody against P53 (1:100, ab26, Abcam, China), and rabbit monoclonal antibody against Bax (1:250, ab32503, Abcam, China) at $4^{\circ} \mathrm{C}$ overnight. The sections were subsequently incubated with Sheep Anti-Mouse IgG H\&L secondary antibody (Texas Red) (1:500, ab6806, Abcam, China), Donkey Anti-Goat IgG H\&L (Alexa Fluor 647) (1:200, ab150135, Abcam, China), and Alexa Fluor 488 Monkey Anti-Rabbit IgG (H + L) antibody (1:1000, A21206, Life Technologies, Beijing, China) at room temperature for $2 \mathrm{~h}$. Subsequently, the sections were incubated with the DAPI dyeing kit (1:500, C0060, Solarbio, Beijing, China) for $15 \mathrm{~min}$. The sections were covered with coverslips for microscopic observation after antibody incubation. Images were acquired using a Nikon Eclipse (TE2000-E) inverted C1 confocal microscope (Nikon Instruments, Minato, Tokyo, Japan) equipped with an oil immersion 60x objective with 1.4 numerical aperture (Nikon) or a Zeiss video microscope (Zeiss AG, Oberkochen, Germany) equipped with a plan Neofluar 10x/0.3 numerical aperture. Antibodies of Bax, P53, caspase-3, and DAPI were used for the triple labeling of apoptosis.

2.9. Enzyme-Linked Immunosorbent Assay. The contents of the tumor necrosis factor- $\alpha$ (TNF- $\alpha$ ), interleukin-1 $\beta$ (IL$1 \beta$ ), interleukin-6 (IL-6), vascular cell adhesion molecule 1 (VCAM-1), and intercellular adhesion molecule 1 (ICAM1) of the supernatant were detected by a sandwich enzymelinked immunosorbent assay (ELISA) according to the manufacturer's protocol. Absorbances were measured at $495 \mathrm{~nm}$ using a microplate ELISA reader (Bio-Rad Model 680 microplate reader, Multiskan FC, Thermo Fisher Scientific Oy, Vantaa, Finland). Each final value was quantified against a standard curve calibrated with known amounts of protein. 


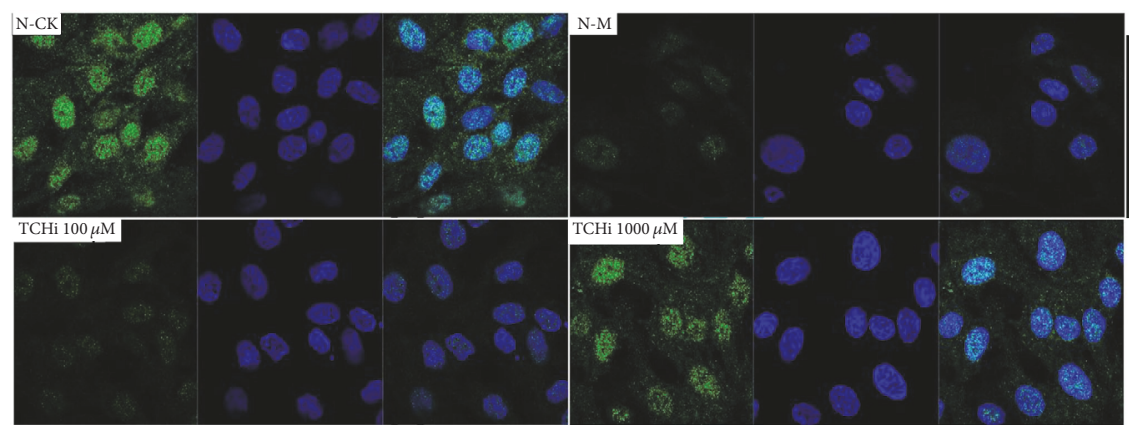

(a)

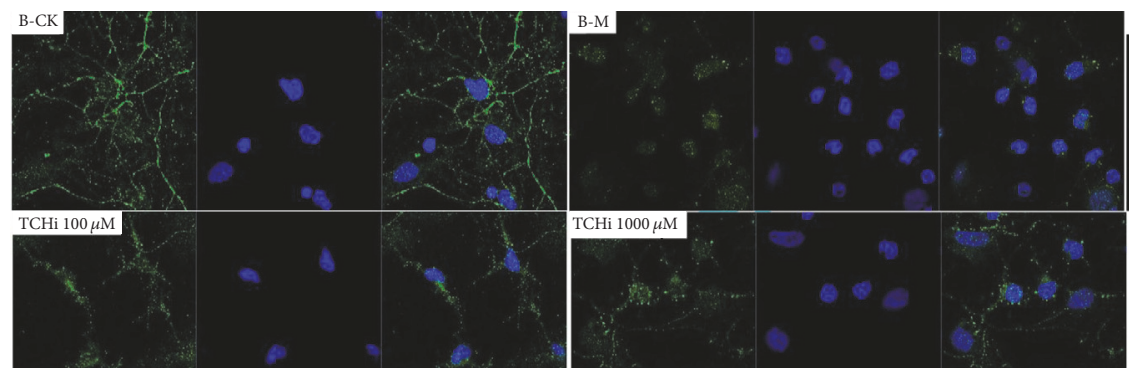

(b)

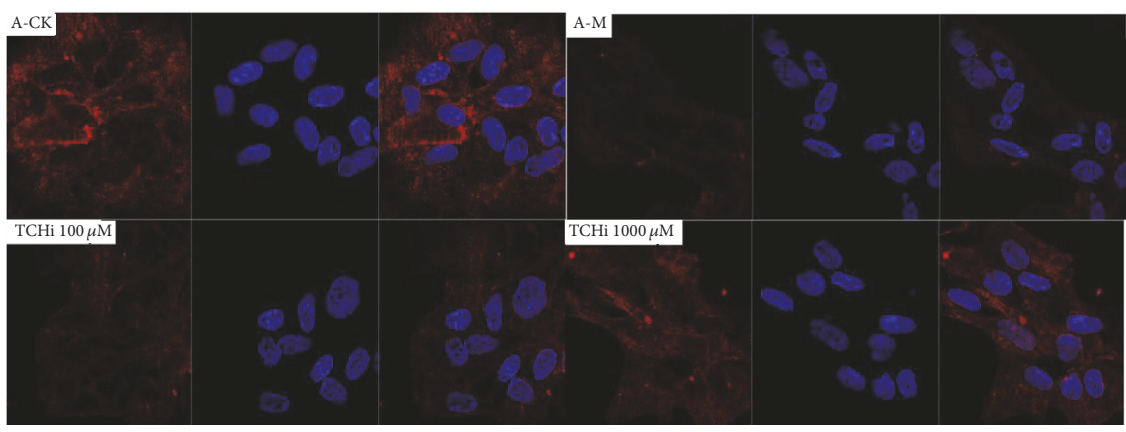

(c)
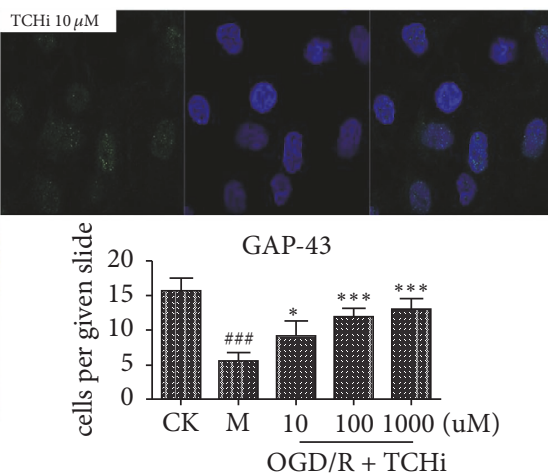

TCHi $10 \mu \mathrm{M}$
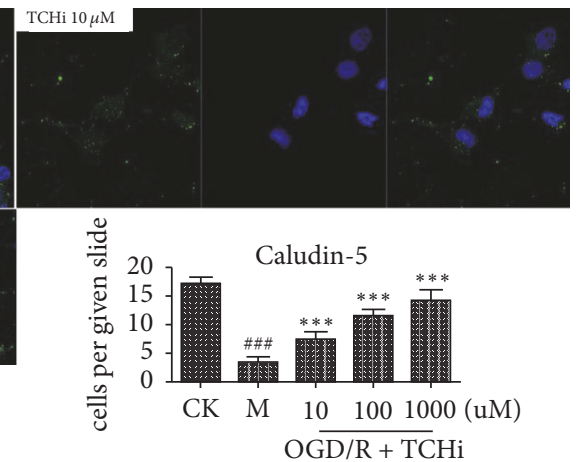

$\mathrm{OGD} / \mathrm{R}+\mathrm{TC} H \mathrm{i}$
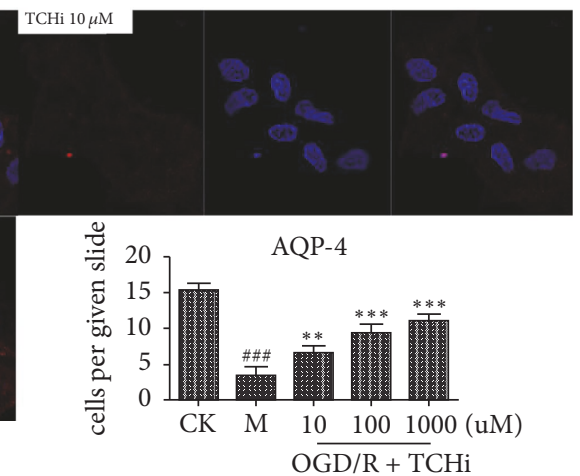

FIGURE 4: Immunocytochemical analysis demonstrated that TCHi had effects on NVU cells against OGD/R. (a), (b), and (c) reflect neurons, rBMECs, and astrocytes, respectively (GAP-43 is labeled in green in (a), Claudin-5 is labeled in green in (b), AQP-4 is labeled in red in (c), and DAPI is labeled in blue). ${ }^{*} P<0.05,{ }^{* *} P<0.01$, and ${ }^{* * *} P<0.001$ relative to Model; ${ }^{\# \# \#} P<0.01$ relative to CK.

2.10. Statistical Analysis. All the results were repeated at least three times. All pictures were analyzed by Adobe Photoshop software. Data were statistically analyzed by analysis of variance (using IBM SPSS 17.0). Results were expressed as mean \pm SD. $P<0.05$ was regarded to be statistically significant.

\section{Result}

Morphology and specific identification for three types of cells in the coculture system: as shown in Figure 2, pictures of the same field were obtained in visible light under the fluorescent inverted microscope. Neuronal cells were cultured at the bottom of the Transwell filter (Figure 2(a)), rBMECs (Figure 2(b)) were seeded on the upper side of the Transwell filter of the inserts, and astrocytes were seeded on the opposite side of the Transwell filter of the inserts (Figure 2(c)). The schematic drawing of the triple cell coculture system is shown in Figure 1. The transendothelial electrical resistance (TEER) of different models indicated that the coculture system had an acceptable BBB function (Figure 2(d)). TEM findings demonstrated that BBB appeared normal in rBMEC; meanwhile, tight junctions and desmosomes were close and adjacent (Figure 3 ). 


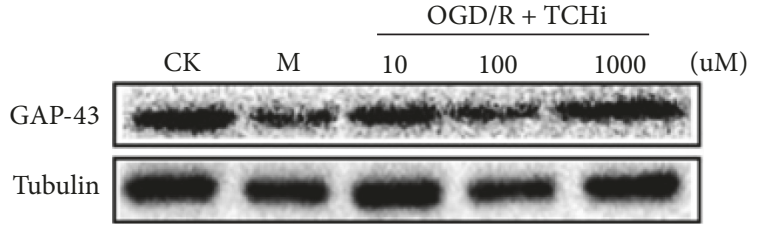

(a)

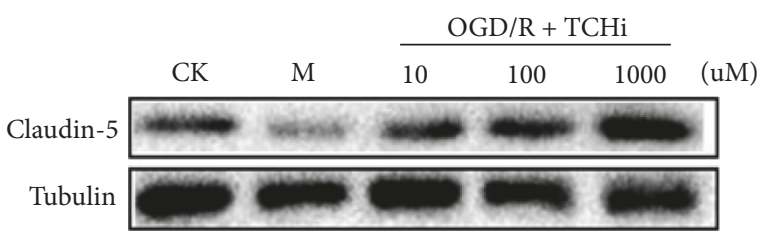

(c)

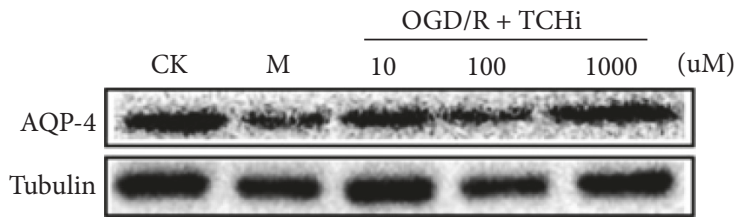

(e)

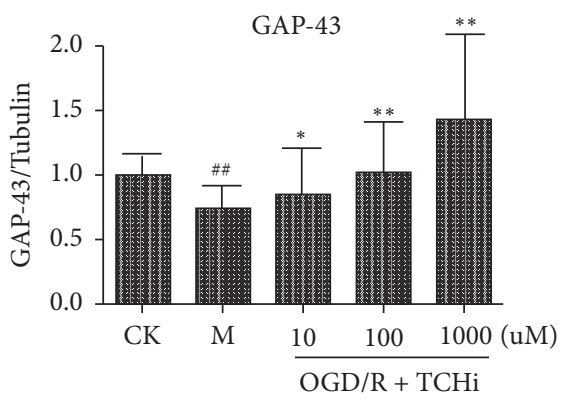

(b)

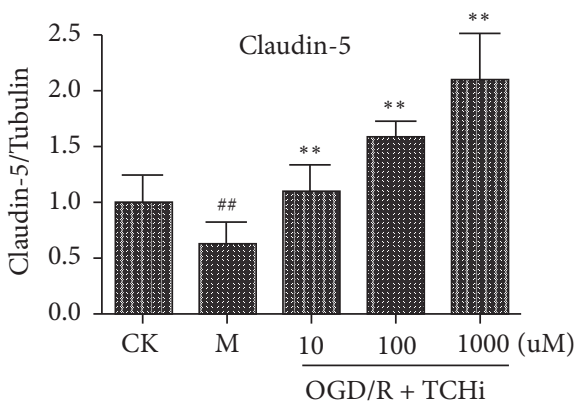

(d)

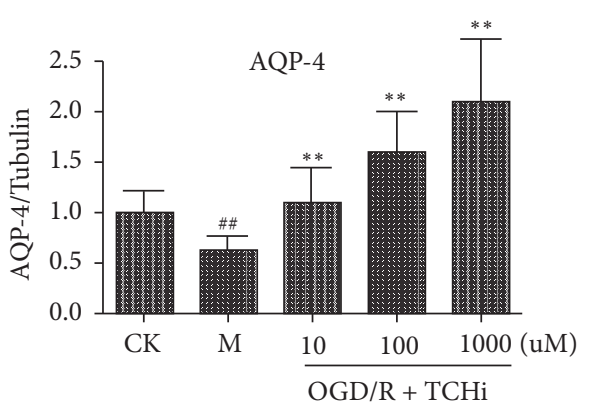

(f)

FIGURE 5: Western blotting showed that TCHi had effects on NVU cells against OGD/R. (a) and (b) are bands of GAP-43 and, on a comparison of densities, indicate that TCHi at concentrations of $10 \mu \mathrm{M}, 100 \mu \mathrm{M}$, and $1000 \mu \mathrm{M}$ maintains neurons after OGD/R. (c) and (d) are bands of Claudin-5 and, on a comparison of densities, indicate that TCHi at concentrations of $10 \mu \mathrm{M}, 100 \mu \mathrm{M}$, and $1000 \mu \mathrm{M}$ maintains rBMECs after OGD/R. (e) and (f) are bands of AQP-4 and, on a comparison of densities, indicate that TCHi at concentrations of $10 \mu \mathrm{M}, 100 \mu \mathrm{M}$, and $1000 \mu \mathrm{M}$ maintains astrocytes after OGD/R.

3.1. Effects of TCHi on Cell Survival in NVU Cells after OGD/R. As shown in Figure 4, neurons, astrocytes, and rBMECs had typical damage manifestations after OGD/R. However, with the treatment of TCHi at doses of $10 \mu \mathrm{M}, 100 \mu \mathrm{M}$, and $1000 \mu \mathrm{M}$, these manifestations were weakened. Western blotting was analyzed to confirm the effect of TCHi, and the findings were similar to those of immunocytochemical analysis (Figures 4 and 5).

3.2. Effects of TCHi on Inflammatory Cytokines in the NVU Model after OGD/R. OGD/R-induced inflammation was inhibited by TCHi in the NVU model. As shown in Table 2, IL-1 $\beta$, IL-6, and TNF- $\alpha$ levels decreased significantly in TCHi $10 \mu \mathrm{M}(P<0.01)$ and TCHi $100 \mu \mathrm{M}(P<0.01)$. Apart from IL$1 \beta$ and IL-6 $(P<0.01)$, TCHi $1000 \mu \mathrm{M}$ showed a more obvious effect on TNF- $\alpha$ levels $(P<0.001)$.
As well as regulating inflammatory cytokines, cell adhesion molecules, such as vascular cell adhesion molecule 1 (VCAM-1) and intercellular adhesion molecule 1 (ICAM1), which have the potential to recruit peripheral leukocytes and other cytokines, were upregulated by OGD/R. ELISA results (Table 1) demonstrated that TCHi $10 \mu \mathrm{M}(P<0.05)$, TCHi $100 \mu \mathrm{M}(P<0.05)$, and TCHi $1000 \mu \mathrm{M}(P<0.05)$ ameliorated the increase in the VACM-1 level significantly. The inhibitory effect on ICAM-1 seemed even more apparent in TCHi $1000 \mu \mathrm{M}(P<0.01)$.

3.3. Effects of TCHi on Antiapoptosis in the NVU Model after $O G D / R$. Whether TCHi had effects on apoptosis was further checked. Proapoptotic factors, Bax, p53, and caspase-3, were detected using immunocytochemical analysis. Figure 6 showed that TCHi at concentrations of $10 \mu \mathrm{M}, 100 \mu \mathrm{M}$, and $1000 \mu \mathrm{M}$ suppressed all these factors. 
TABLE 1: Expression of cell adhesion molecules in the cell culture medium of NVU (mean $\pm \mathrm{SD}, N=6)$.

\begin{tabular}{lcr}
\hline Groups & VCAM-1 $(\mathrm{pg} / \mathrm{mL})$ & ICAM-1 $(\mathrm{pg} / \mathrm{mL})$ \\
\hline CK & $29.03 \pm 2.31$ & $21.69 \pm 4.40$ \\
Model & $142.46 \pm 6.33^{\# \# \#}$ & $90.31 \pm 15.63^{\# \# \#}$ \\
TCHi $10 \mu \mathrm{M}$ & $119.88 \pm 9.17^{*}$ & $73.44 \pm 14.61^{*}$ \\
TCHi $100 \mu \mathrm{M}$ & $104.94 \pm 4.13^{*}$ & $45.19 \pm 22.69^{* *}$ \\
TCHi $1000 \mu \mathrm{M}$ & $104.19 \pm 4.81^{*}$ & $38.97 \pm 7.46^{* *}$ \\
\hline
\end{tabular}

${ }^{*} P<0.05$ and ${ }^{* *} P<0.01$ relative to Model; ${ }^{\# \#} P<0.001$ relative to CK.

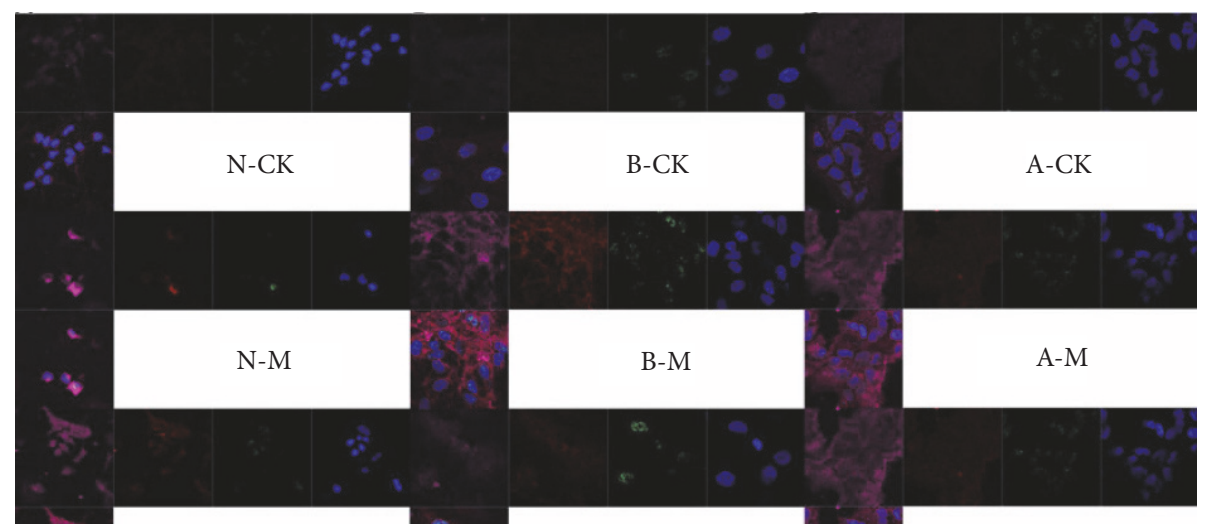

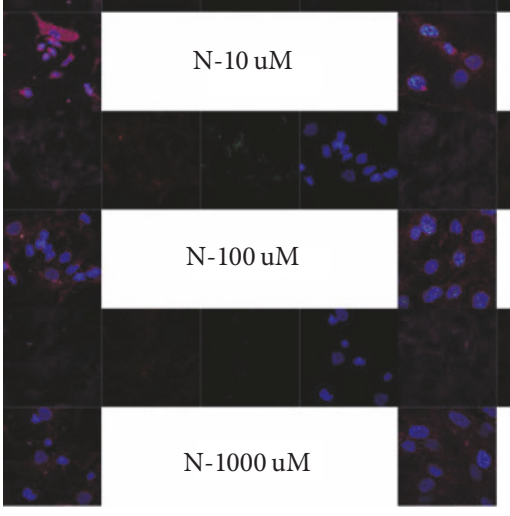

(a)

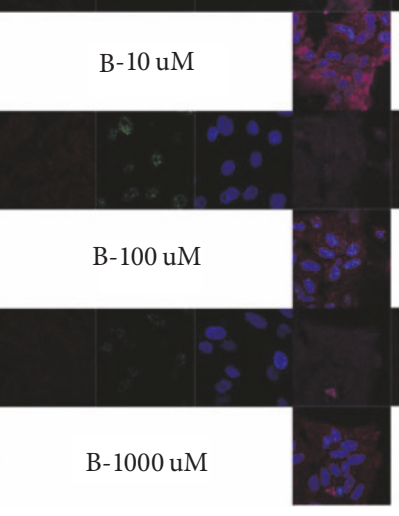

(b)
A-10 uM

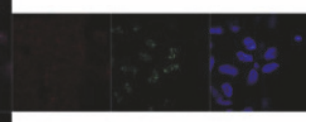

A-100 uM

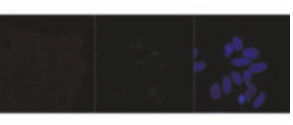

A-1000 uM

(c)

FiguRE 6: Immunocytochemical analysis demonstrated that TCHi had antiapoptotic effects against OGD/R. (a), (b), and (c) reflect neurons, rBMECs, and astrocytes, respectively (Bax is labeled in orange, p53 is labeled in red, caspase-3 is labeled in green, and DAPI is labeled in blue).

3.4. Effects of TCHi on the Ultrastructure of NVU Cells after $O G D / R$. The ultrastructures of neurons and rBMECs were observed by TEM to confirm the effectiveness of TCHi. Figure 7 indicated that both neurons and rBMECs showed apoptotic signs after OGD/R. Cells exhibited shrinkage of shape, irregular nuclei, diffused distribution of heterochromatin, autophagosome appearance, and so on. At concentrations of $1000 \mu \mathrm{M}$, TCHi reversed all these signs.

\section{Discussion}

Our study demonstrated that an injection of TCH could ameliorate OGD/R, inducing NVU dysfunction. The mechanism of TCH treatment was to suppress inflammation and apoptosis. All these findings in the NVU model implied the underlying therapeutic effect of TCHi against cerebral ischemic strokes, which might explain positive clinical findings for TCHi [9].

Neuroinflammation is a complex inflammatory process in the central nervous system, which is thought to play an important defensive role against various pathogens [15]. However, an aberrant inflammatory response is known to be a type of reperfusion injury after a stroke [16]. Several cytokines and chemokines are released after an ischemic brain injury. The most extensively studied of the proinflammatory cytokines include IL- $1 \beta$, IL- 6 , and TNF- $\alpha$ [16]. This study measured the concentrations of IL- $1 \beta$, IL- 6 , and TNF- $\alpha$ in the OGD/R model and found that TCHi has an inhibitory effect on all these cytokines.

An inflammatory process consists of both the activation of resident cells of the central nervous system and the infiltration of peripheral leukocytes into the ischemic 
TABLE 2: Expression of cytokines in the cell culture medium of NVU (mean $\pm \mathrm{SD}, N=6$ ).

\begin{tabular}{lccc}
\hline Groups & IL-1 $\beta(\mathrm{pg} / \mathrm{mL})$ & IL-6 $(\mathrm{pg} / \mathrm{mL})$ & TNF- $\alpha(\mathrm{pg} / \mathrm{mL})$ \\
\hline CK & $280.09 \pm 13.36$ & $30.07 \pm 5.81$ & $29.56 \pm 3.57$ \\
Model & $568.13 \pm 33.36^{\# \# \#}$ & $433.01 \pm 20.56^{\# \#}$ & $156.49 \pm 17.98^{\# \# \#}$ \\
TCHi $10 \mu \mathrm{M}$ & $485.57 \pm 27.18^{* *}$ & $387.44 \pm 30.66^{*}$ & $88.43 \pm 7.00^{* *}$ \\
TCHi $100 \mu \mathrm{M}$ & $446.92 \pm 18.57^{* *}$ & $305.90 \pm 22.69^{* *}$ & $85.51 \pm 2.99^{* *}$ \\
TCHi $1000 \mu \mathrm{M}$ & $314.29 \pm 20.45^{* *}$ & $289.75 \pm 17.49^{* *}$ & $36.06 \pm 3.48^{* * *}$ \\
\hline
\end{tabular}

${ }^{*} P<0.05,{ }^{* *} P<0.01$, and ${ }^{* * *} P<0.001$ relative to Model; ${ }^{\# \#} P<0.01$ and ${ }^{\# \#} P<0.01$ relative to $C K$.

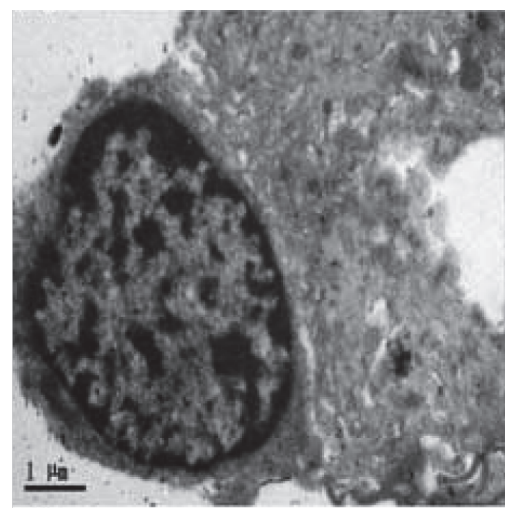

(a)

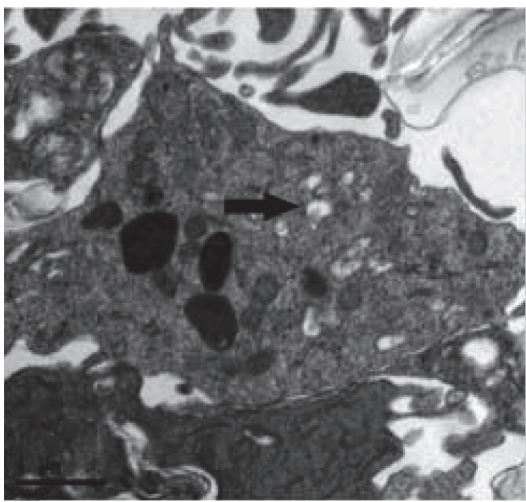

(d)

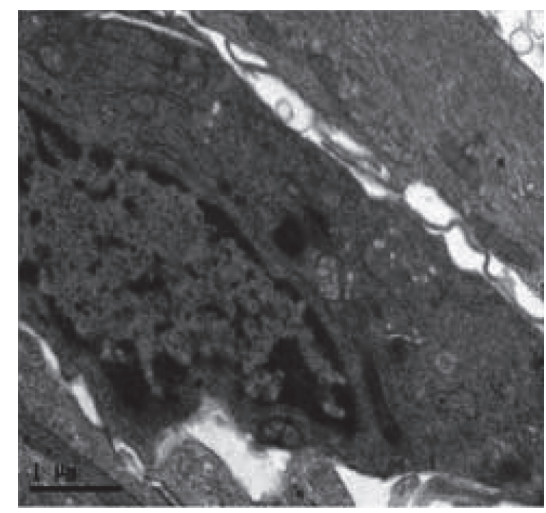

(b)

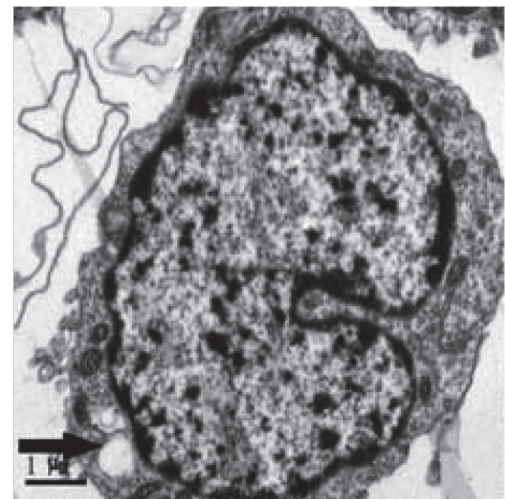

(e)

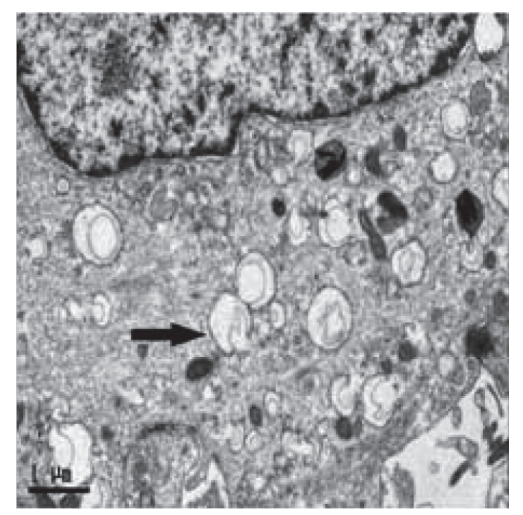

(c)

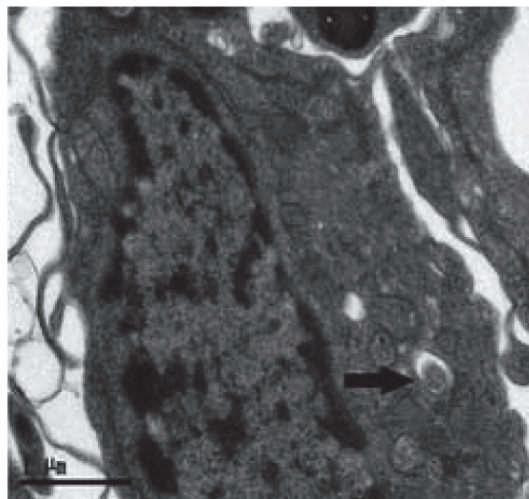

(f)

FIGURE 7: TEM indicated that TCHi alleviated the abnormalities of ultrastructures of neurons and rBMECs. (a) and (b) show that, in normal condition, both neurons and rBMECs exhibit a normal shape and a regular nucleus. (c) and (d) reveal that both neurons and rBMECs show shrinkage of shape, irregular nucleus, diffused distribution of heterochromatin, and amount of autophagosome appearance (black arrow) after OGD/R. (e) and (f) demonstrate that $1000 \mu \mathrm{M}$ TCHi could reverse the apoptotic appearances of cells.

brain tissue [17]. Cell adhesion molecules are involved in the trafficking and recruitment of leukocytes to activate ischemic endothelia in strokes, which could worsen ischemic brain injuries [18]. ICAM-1 and VCAM-1 are reported to be upregulated by the proinflammatory cytokines TNF- $\alpha$ and IL-1 $\beta[19,20]$. The results of the present study revealed that the anti-inflammatory properties of TCHi in an OGD/Rdamaged NVU model were due to the reducing levels of ICAM-1 and VCAM-1 as well as cytokines.

Apart from inflammation, apoptosis is another focus in the pathogenesis of brain injuries [17]. The regulation of the apoptosis process is essential to maintain the balance between cell survival and cell death, which is also important in ischemic injuries [21]. Proapoptotic proteins, such as Bax, $\mathrm{p} 53$, and caspase-3, were reported to take part in I/R injuries earlier [21]. The current study demonstrated that TCHi could be an effective therapy against apoptosis. It has an impact on the regulation of the apoptosis process via decreasing Bax, p53, and caspase-3 levels in an OGD/R-damaged NVU model.

Not only is BBB the key component of NVU, but also it is the most important structure to maintain cerebral homeostasis and correct neuronal function [22]. BBB integrity in the present study was reflected in TEER, tight junctions, and other ultrastructures of NVUs. TCHi was found to alleviate the BBB breakdown in the OGD/R model. Previous 
researchers have confirmed the anti-inflammatory and antiapoptotic roles of troxerutin in chronic diseases and diabetic models $[23,24]$. The findings of this study revealed that $\mathrm{TCHi}$, a new compound of troxerutin, had a good therapeutic effect in acute attacks such as cerebral I/R injuries.

This study still has limitations. Inflammatory cytokines and cell adhesion molecules were found to be upregulated in NVUs for the in vitro OGD/R model; however, the infiltration of peripheral leukocytes and other factors in the central nervous system could not be demonstrated. As a result, further in vivo studies should be performed to show whether TCHi had the same effects as in the in vitro model.

\section{Conclusion}

TCHi protected the main types of cells of NVUs in vivo and in vitro depending on anti-inflammation, antiapoptosis, and $\mathrm{BBB}$. The data implies that TCHi is a candidate medicine to treat cerebral ischemic stroke.

\section{Disclosure}

Hóngyi Zhào and Yu Liu are co-first authors in this article.

\section{Conflicts of Interest}

The authors declare that they have no conflicts of interest.

\section{Acknowledgments}

The authors thank Professor Xiaoyu Xu and M.D. Qiang Xue for their technical support for the in vitro NVU model.

\section{References}

[1] D. J. Hausenloy and D. M. Yellon, "Ischaemic conditioning and reperfusion injury," Nature Reviews Cardiology, vol. 13, no. 4, pp. 193-209, 2016.

[2] M. M. Khan, C. Gandhi, N. Chauhan et al., "Alternativelyspliced extra domain a of fibronectin promotes acute inflammation and brain injury after cerebral ischemia in mice," Stroke, vol. 43, no. 5, pp. 1376-1382, 2012.

[3] S. S. Raza, M. M. Khan, A. Ahmad et al., "Neuroprotective effect of naringenin is mediated through suppression of NF$\kappa \mathrm{B}$ signaling pathway in experimental stroke," Neuroscience, vol. 230, pp. 157-171, 2013.

[4] V. Muoio, P. B. Persson, and M. M. Sendeski, "The neurovascular unit-concept review," Acta Physiologica, vol. 210, no. 4, pp. 790-798, 2014.

[5] G. J. del Zoppo, "The neurovascular unit in the setting of stroke," Journal of Internal Medicine, vol. 267, no. 2, pp. 156-171, 2010

[6] X. Tian, J. Peng, J. Zhong et al., " $\beta$-Caryophyllene protects in vitro neurovascular unit against oxygen-glucose deprivation and re-oxygenation-induced injury," Journal of Neurochemistry, vol. 139, no. 5, pp. 757-768, 2016.

[7] F. Liu, Y. Huang, and P. Fu, "Troxerutin Counteracts domoic acidinduced memory deficits in mice by inhibiting CCAAT/ Enhancer binding protein $B$-mediated inflammatory response and oxidative stress," Journal of Immunology, vol. 190, pp. 34663479, 2013.
[8] B. Mokhtari, R. Badalzadeh, A. Alihemmati, and M. Mohammadi, "Phosphorylation of GSK-3 $\beta$ and reduction of apoptosis as targets of troxerutin effect on reperfusion injury of diabetic myocardium," European Journal of Pharmacology, vol. 765, pp. 316-321, 2015.

[9] H. Chen, K. Liang, s. Zhou, F. Hu, M. Te, and H. Lu, "Curative effect and security of troxerutin and cerebroprotein hydrolysate injection in acute cerebral infarction, a multicenter,randomized,single blind and placebo control study," Chinese Journal of Neuroimmunology \& Neurology, vol. 23, pp. 251-255, 2016.

[10] Q. Xue, Y. Liu, H. Qi et al., "A novel brain neurovascular unit model with neurons, astrocytes and microvascular endothelial cells of rat," International Journal of Biological Sciences, vol. 9, no. 2, pp. 174-189, 2013.

[11] M. Wang, D. Zhong, Y. Zheng et al., "Damage effect of interleukin (IL)-23 on oxygen-glucose-deprived cells of the neurovascular unit via IL-23 receptor," Neuroscience, vol. 289, pp. 406-416, 2015.

[12] Q. Xue, Y. Liu, R. He et al., "Lyophilized powder of catalpol and puerarin protects neurovascular unit from stroke," International Journal of Biological Sciences, vol. 12, no. 4, pp. 367-380, 2016.

[13] R. Liu, T.-T. Zhang, C.-X. Wu, X. Lan, and G.-H. Du, “Targeting the neurovascular unit: Development of a new model and consideration for novel strategy for Alzheimer's disease," Brain Research Bulletin, vol. 86, no. 1-2, pp. 13-21, 2011.

[14] S. Garbuzova-Davis, E. Haller, S. Saporta, I. Kolomey, S. V. Nicosia, and P. R. Sanberg, "Ultrastructure of blood-brain barrier and blood-spinal cord barrier in SOD1 mice modeling ALS," Brain Research, vol. 1157, no. 1, pp. 126-137, 2007.

[15] A. Tohidpour, A. V. Morgun, E. B. Boitsova et al., "Neuroinflammation and infection: Molecular mechanisms associated with dysfunction of neurovascular unit," Frontiers in Cellular and Infection Microbiology, vol. 7, no. 276, 2017.

[16] A. Mizuma and M. A. Yenari, "Anti-inflammatory targets for the treatment of reperfusion injury in stroke," Frontiers in Neurology, vol. 8, article no. 467, 2017.

[17] N. Quillinan, P. S. Herson, and R. J. Traystman, "Neuropathophysiology of brain injury," Anesthesiology Clinics, vol. 34, no. 3, pp. 453-464, 2016.

[18] T. Yoshimoto, K. Houkin, M. Tada, and H. Abe, "Induction of cytokines, chemokines and adhesion molecule mRNA in a rat forebrain reperfusion model," Acta Neuropathologica, vol. 93, no. 2, pp. 154-158, 1997.

[19] C. J. M. Frijns and L. J. Kappell, "Inflammatory cell adhesion molecules in ischemic cerebrovascular disease," Stroke, vol. 33, no. 8, pp. 2115-2122, 2002.

[20] R. L. Zhang, M. Chopp, N. Jiang et al., "Anti-intercellular adhesion molecule-1 antibody reduces ischemic cell damage after transient but not permanent middle cerebral artery occlusion in the Wistar rat," Stroke, vol. 26, no. 8, pp. 1438-1443, 1995.

[21] C. H. Chen, Z. Jiang, J. H. Yan et al., "The involvement of programmed cell death 5 (PDCD5) in the regulation of apoptosis in cerebral ischemia/reperfusion injury," Cns Neuroscience \& Therapeutics, vol. 19, pp. 566-576, 2013.

[22] A.-C. Luissint, C. Artus, F. Glacial, K. Ganeshamoorthy, and P.-O. Couraud, "Tight junctions at the blood brain barrier: physiological architecture and disease-associated dysregulation," Fluids and Barriers of the CNS, vol. 9, no. 1, article 23, 2012.

[23] J. Lu, D.-M. Wu, Y.-L. Zheng et al., "Troxerutin counteracts domoic acid-induced memory deficits in mice by inhibiting 
CCAAT/enhancer binding protein $\beta$-mediated inflammatory response and oxidative stress," The Journal of Immunology, vol. 190, pp. 3466-3479, 2013.

[24] R. Yavari, R. Badalzadeh, M. R. Alipour, and S. M. Tabatabaei, "Modulation of hippocampal gene expression of microRNA146a/microRNA-155-nuclear factor-kappa B inflammatory signaling by troxerutin in healthy and diabetic rats," Indian Journal of Pharmacology, vol. 48, no. 6, pp. 675-680, 2016. 


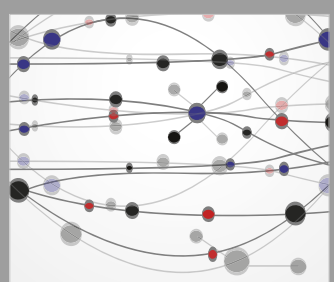

The Scientific World Journal
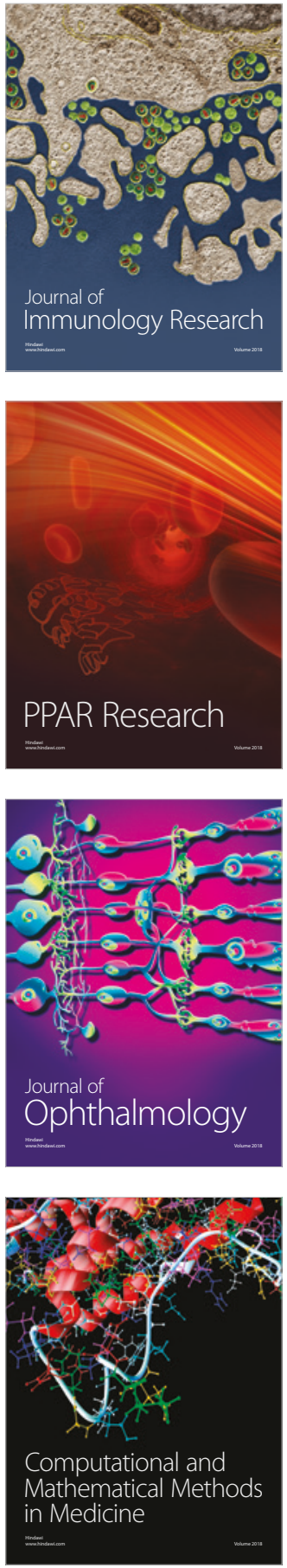

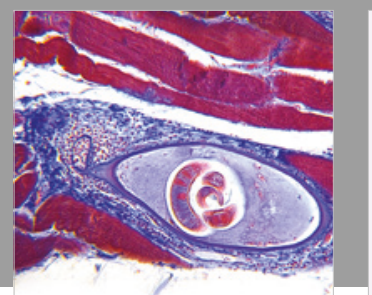

Gastroenterology Research and Practice

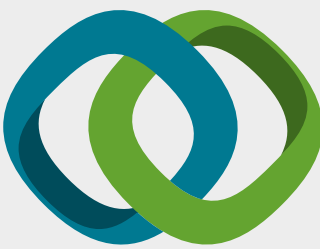

\section{Hindawi}

Submit your manuscripts at

www.hindawi.com
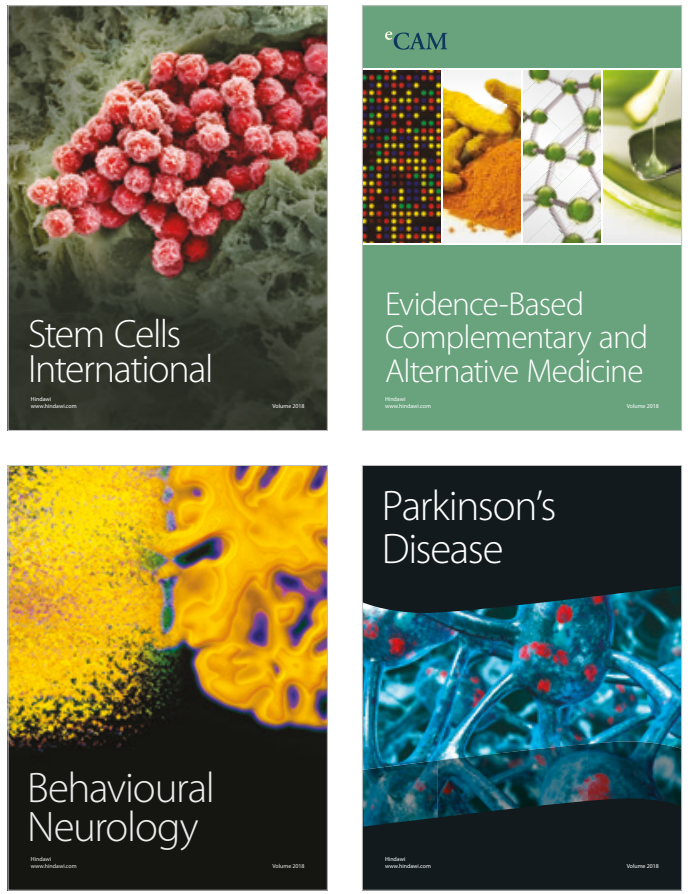

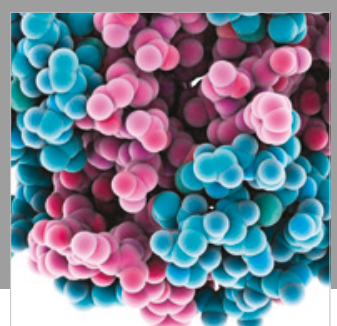

ournal of

Diabetes Research

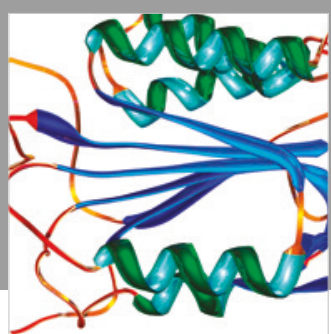

Disease Markers
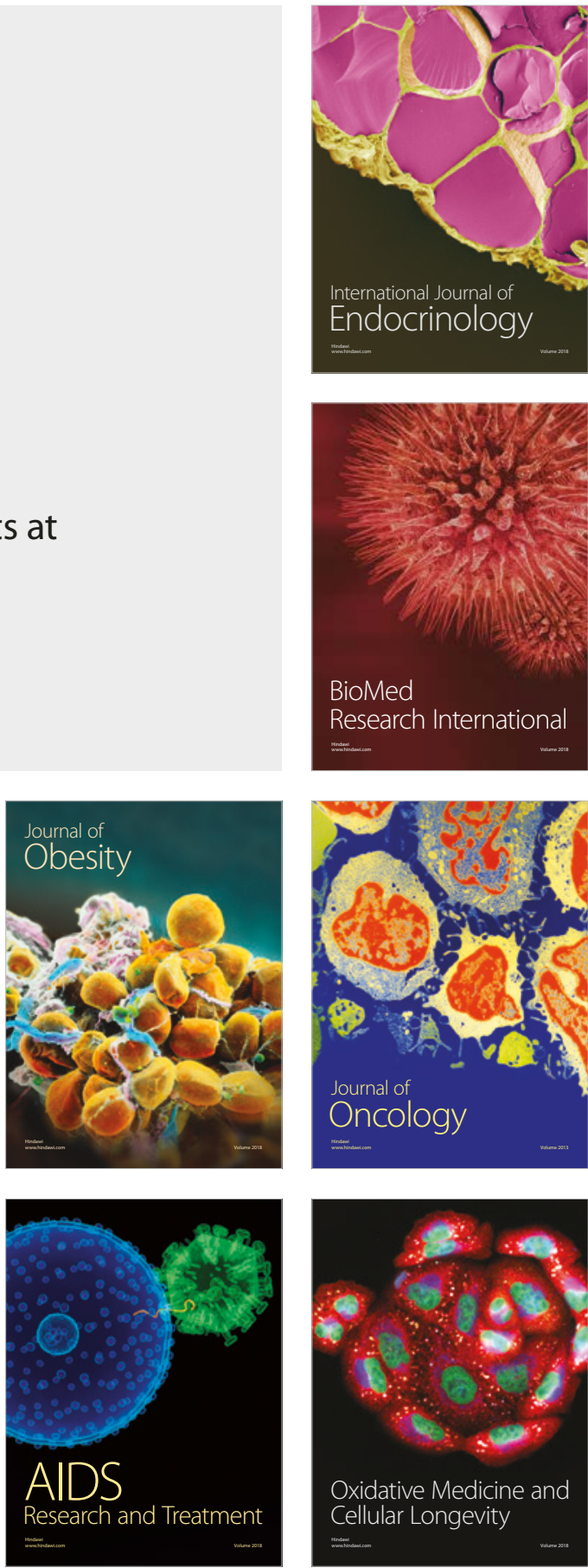